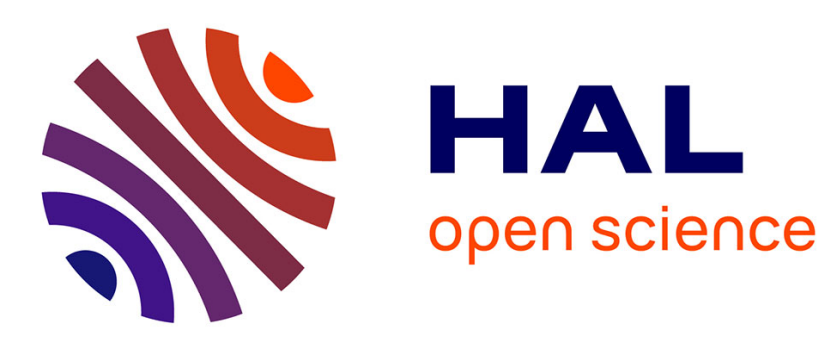

\title{
The Propagation Characteristics of 2-D Metamaterial Waveguides Using the Modal Expansion Theory
}

\author{
Lucille Kuhler, Gwenn Le Fur, Luc Duchesne, Nathalie Raveu
}

\section{To cite this version:}

Lucille Kuhler, Gwenn Le Fur, Luc Duchesne, Nathalie Raveu. The Propagation Characteristics of 2-D Metamaterial Waveguides Using the Modal Expansion Theory. IEEE Transactions on Microwave Theory and Techniques, 2018, 66 (10), pp.4319-4326. 10.1109/TMTT.2018.2859944 . hal-02439737

\section{HAL Id: hal-02439737 \\ https://hal-enac.archives-ouvertes.fr/hal-02439737}

Submitted on 14 Jan 2020

HAL is a multi-disciplinary open access archive for the deposit and dissemination of scientific research documents, whether they are published or not. The documents may come from teaching and research institutions in France or abroad, or from public or private research centers.
L'archive ouverte pluridisciplinaire HAL, est destinée au dépôt et à la diffusion de documents scientifiques de niveau recherche, publiés ou non, émanant des établissements d'enseignement et de recherche français ou étrangers, des laboratoires publics ou privés. 


\title{
The Propagation Characteristics of 2D Metamaterial Waveguides Using the Modal Expansion Theory
}

\author{
Lucille Kuhler, Gwenn Le Fur, Luc Duchesne and Nathalie Raveu
}

\begin{abstract}
In this article, the Modal Expansion Theory is applied to 2D metamaterial cylindrical waveguides. A new code to compute the surface impedances with 2D Finite Element Method is put forward. The surface impedances depend on the frequency and the incidence angle. The characterization of propagation in a $2 \mathrm{D}$ metamaterial waveguide is presented by using the Modal Expansion Theory and 2D Finite Element Method. Comparisons with the software HFSS are carried out in order to validate the new method.
\end{abstract}

Index Terms-Anisotropic surface impedances, cylindrical waveguides, dispersion diagrams, metamaterials, Modal Expansion Theory

\section{INTRODUCTION}

$\mathbf{M}$ ETAMATERIALS are widely used in industry. They allow creating materials with electromagnetic properties that are not available in natural materials [1]. Their main property arises from their structuration rather than their composition [2]. Concerning their structuration, they are periodically set, and their period is small compared to the wavelength [2]. Thanks to their properties, it is possible to obtain a relative permittivity and/or a permeability lower than one or less than zero [2]-[4]. In research metamaterials are mostly defined by characterizing both permittivity and permeability. The other method of characterizing metamaterials is by considering their surface impedances at any given volume height [5].

Using metamaterials in waveguides or in horn antennas can reduce their size and their weight [6]-[9]. This effect is fundamental in the space industry, decreasing the mass of spacecraft devices lowers the launch cost [10]-[12]. To optimize the metamaterial in such devices, the balanced hybrid

Manuscript submitted February 2, 2018; revised April 11, 2018. This work was funded by the CNES and made in collaboration between the CNES, MVG Industries and the LAPLACE laboratory.

L. Kuhler is with the University of Toulouse, INPT, UPS, LAPLACE, ENSEEIHT, Toulouse 31071, France, in collaboration with the Centre National d'Etude Spatiale (CNES), Toulouse 31400, France, and also with MVG Industries, Villebon-Sur-Yvette 91140, France (e-mail: lucille.kuhler@laplace.univ-tlse.fr)

G. LeFur is with the Centre National d'Etude Spatiale (CNES), Toulouse 31400, France (e-mail: gwenn.lefur@cnes.fr)

L. Duchesne is with MVG Industries, Villebon-Sur-Yvette 91140, France (e-mail: luc.duchesne@microwavevision.com)

N. Raveu is with the University of Toulouse, INPT, UPS, LAPLACE, ENSEEIHT, Toulouse 31071, France (e-mail: raveu @laplace.univ-tlse.fr). condition [13] is commonly used. However this method of optimization is time-consuming since commercial software is based on 3D meshing. Moreover no proof is given that this condition is necessary. In [14] the cross-section of a rectangular waveguide has been reduced without this condition.

In previous work [15]-[17] the Modal Expansion Theory (MET) has been developed for cylindrical and rectangular waveguides with constant anisotropic surface impedances. This method allows a fast computation of waveguide properties. However real metamaterials have to be taken into account. In [18] real 2D metamaterials in rectangular waveguides have been studied with surface impedances depending on the frequency and incidence angle.

In this article $2 \mathrm{D}$ metamaterial waveguides are characterized with the cylindrical MET. The surface impedances defined at any given volume height are computed thanks to a conformal 2D Finite Element Method (FEM) developed in Section II. In this Section the propagation equation in a cylindrical waveguide is recalled as well as its recursive dependence on the incidence angle. In the final part the method validation is presented by comparing the dispersion diagrams obtained with HFSS. Three different waveguides are studied, the first is a waveguide with fixed surface impedances, the second is a corrugated waveguide and the last is a cylindrical waveguide with a T-structure metamaterial.

\section{CHARACTERIZATION PRINCIPLES}

In this article, a cylindrical waveguide with metamaterial walls is considered invariant along the $z$-axis, see Fig. 1. Therefore the electromagnetic field has an $e^{-\gamma z}$ dependence, with $\gamma$ the propagation constant along the $z$-axis.

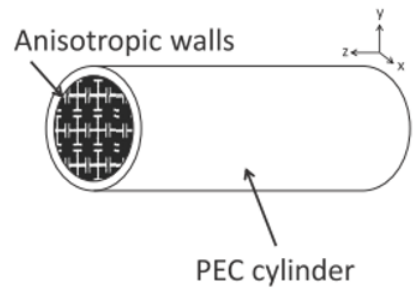

Fig. 1. Cylindrical waveguide with anisotropic walls.

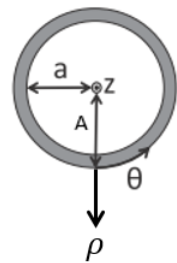

$\rho$ 
The MET is proposed to characterize a cylindrical waveguide with metamaterial walls. As metamaterial periodicity is supposed to be small compared to wavelength, they are assumed to be equivalent to anisotropic surface impedances.

\section{A. Modal Expansion Theory for Cylindrical Waveguides}

In the cylindrical coordinate system, the $Z_{s}^{\prime}$ surface impedance is defined as:

$$
\vec{E}_{T}=Z_{S}^{\prime}\left(\vec{H}_{T} \times \vec{n}\right)
$$

where $\vec{E}_{T}$ and $\vec{H}_{T}$ are the electric and magnetic fields tangent to the cylinder surface, $\vec{n}$ the normal unit vector to the cylinder surface.

Usually in the cartesian coordinate system, $Z_{T}^{\prime}$ stands for the TE modes impedance and $Z_{Z}^{\prime}$ for the TM modes impedance. In cylindrical coordinates, they are defined by:

$$
Z_{T}^{\prime}=-\left.\frac{E_{\theta}}{H_{Z}}\right|_{\rho=a}, \quad Z_{Z}^{\prime}=\left.\frac{E_{Z}}{H_{\theta}}\right|_{\rho=a} .
$$

The dispersion equation (3) is determined in [16] from Helmholtz's equation and the anisotropic boundary conditions.

$$
\begin{gathered}
\frac{Z_{Z}^{\prime}}{Z_{0}}\left(J_{m}^{\prime}\left(u_{a}\right)\right)^{2}-\frac{Z_{T}^{\prime}}{Z_{0}}\left(\frac{k_{c} J_{m}\left(u_{a}\right)}{k_{0}}\right)^{2} \\
+\left(\frac{Z_{Z}^{\prime} Z_{T}^{\prime}}{Z_{0}^{2}}+1\right) \frac{k_{c} J_{m}\left(u_{a}\right) J_{m}^{\prime}\left(u_{a}\right)}{j k_{0}} \\
+\frac{Z_{Z}^{\prime}}{Z_{0}}\left(\left(\frac{k_{c}}{k_{0}}\right)^{2}-1\right)\left(\frac{m J_{m}\left(u_{a}\right)}{u_{a}}\right)^{2}=0
\end{gathered}
$$

where $a$ is the internal radius, $u_{a}=k_{c} a, Z_{0}$ the free space characteristic impedance, $k_{c}$ the cutoff constant, $J_{m}$ the Bessel function of order $m, J_{m}^{\prime}$ the derivative of the Bessel function $J_{m}$ and $k_{0}$ the free space wavenumber.

Equation (3) is simplified for $m=0$ modes [16] into:

$$
\begin{gathered}
{\left[j \omega \mu_{0} J_{0}^{\prime}\left(u_{a}\right)+Z_{T}^{\prime} k_{c} J_{0}\left(u_{a}\right)\right]} \\
\times\left[Z_{Z}^{\prime} j \omega \epsilon_{0} J_{0}^{\prime}\left(u_{a}\right)+k_{c} J_{0}\left(u_{a}\right)\right]=0
\end{gathered}
$$

where $\mu_{0}$ is the free space permeability, $\epsilon_{0}$ the free space permittivity and $\omega$ the angular frequency (by using the convention of electromagnetic fields dependent on $e^{j \omega t}$ ).

Metamaterials are considered as anisotropic surface impedances however their shape should be undertaken. In [18] this method is applied with success to rectangular waveguides with $2 \mathrm{D}$ metamaterials with invariance along the $y$-axis. In this article, the same method is applied to cylindrical waveguides with $2 \mathrm{D}$ metamaterials due to an invariance along the $\theta$-axis. Only $m=0$ modes are of interest in this article.
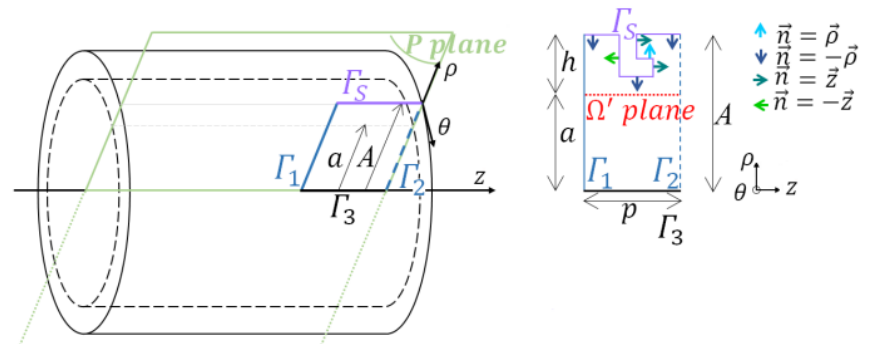

Fig. 2. Cylindrical waveguide with an example of a 2D metamaterial unit cell.

\section{B. The Characterization of Conformal Metamaterials}

In this Section conformal surface impedances are computed with a FEM.

The waveguide is invariant along $\theta$, so the $3 \mathrm{D}$ FEM code is simplified into 2D FEM code. Fig. 2 shows a cylindrical waveguide. In any plane $P$, a unit cell can be extracted as represented in Fig. 2 on the right.

$\Gamma_{1}$ and $\Gamma_{2}$ are periodic boundaries, $\Gamma_{3}$ is the rotational axis and $\Gamma_{S}$ is a boundary where the $Z_{S}$ surface impedance $\left(Z_{T}\right.$ and $Z_{Z}$ ) is defined. $\Omega^{\prime}$ is the plane where the $Z_{S}^{\prime}$ surface impedances $\left(Z_{T}^{\prime}\right.$ and $Z_{Z}^{\prime}$ of (2)) are computed. $A$ is the radius of the waveguide, $h$ is height of the metamaterial and $p$ the distance between $\Gamma_{1}$ and $\Gamma_{2}$.

\section{1) General Formulation}

Each field $\vec{E}$ or $\vec{H}$ is a solution of the propagation equation (5).

$$
\nabla^{2} \vec{U}+k_{0}^{2} \vec{U}=\overrightarrow{0}
$$

where $\vec{U}\left(U_{\rho}, U_{\theta}, U_{z}\right)$ is a vector which could be the magnetic field $\vec{H}$ or the electric field $\vec{E}$.

The $\theta$ component of this equation leads to:

$$
\begin{aligned}
\frac{1}{\rho} \frac{\partial}{\partial \rho}\left(\rho \frac{\partial U_{\theta}}{\partial \rho}\right)+ & \frac{1}{\rho^{2}} \frac{\partial^{2} U_{\theta}}{\partial \theta^{2}}+\frac{\partial^{2} U_{\theta}}{\partial z^{2}}-\frac{U_{\theta}}{\rho^{2}} \\
& +\frac{2}{\rho^{2}} \frac{\partial U_{\rho}}{\partial \theta}+k_{0}^{2} U_{\theta}=0 .
\end{aligned}
$$

As fields are considered $\theta$ invariant $\left(\frac{\partial U_{i}}{\partial \theta}=0\right.$ for $\left.i=\rho, \theta, z\right)$, therefore (6) becomes:

$$
\frac{1}{\rho} \frac{\partial}{\partial \rho}\left(\rho \frac{\partial U_{\theta}}{\partial \rho}\right)+\frac{\partial^{2} U_{\theta}}{\partial z^{2}}+\left(k_{0}^{2}-\frac{1}{\rho^{2}}\right) U_{\theta}=0
$$

The variational formulation [19] of (7) is:

$$
\begin{array}{r}
\iint_{S}\left(\nabla U_{\theta} \cdot \overline{\nabla v}-\left(k_{0}^{2}-\frac{1}{\rho^{2}}\right) U_{\theta} \bar{v}\right) \rho d \rho d z \\
-\int_{\Gamma_{S}} \bar{v} \nabla U_{\theta} \cdot \vec{n} \rho d l=0
\end{array}
$$


where $S$ is the surface of the unit cell, $v(\rho, z)$ a basis function, $\vec{n}$ the normal unit vector to $\Gamma_{s}$, where $\bar{v}$ the $v$ conjugate complex and $d l$ the line element along $\Gamma_{s}$. Along the boundary $\Gamma_{1}$ and $\Gamma_{2}, U_{\theta}$ is considered as follows:

$$
\left.U_{\theta}\right|_{\Gamma_{2}}=\left.U_{\theta}\right|_{\Gamma_{1}} \times \exp \left(-\gamma_{z} p\right)
$$

$\Gamma_{S}$ is divided into $\Gamma_{\text {hor }}$ horizontal walls and $\Gamma_{\text {vert }}$ vertical walls. So:

- $\quad$ for a vertical wall, $\vec{n}= \pm \vec{Z}$ :

$$
\nabla U_{\theta} \cdot \vec{n}= \pm \frac{\partial U_{\theta}}{\partial z}
$$

- $\quad$ for a horizontal wall, $\vec{n}= \pm \vec{\rho}$ :

$$
\nabla U_{\theta} \cdot \vec{n}= \pm \frac{\partial U_{\theta}}{\partial \rho}
$$

(9) and (10) are linked to surface impedances in the following parts.

\section{2) The Characterization of $Z_{Z}^{\prime}$}

$Z_{Z}^{\prime}$ defined in (2) is generated by $H_{\theta}$ which is considered $\theta$ invariant. $H_{\theta}$ is solution of (8).

The last term of (8) is simplified thanks to (10) and (11):

- for vertical walls:

$$
\nabla H_{\theta} \cdot \vec{n}= \pm \frac{\partial H_{\theta}}{\partial z}
$$

with Maxwell's equation and (1):

$$
\frac{1}{j \omega \epsilon_{0}} \nabla \times\left.\vec{H}\right|_{\Gamma_{S}}=Z_{S}\left(\left.\vec{H}\right|_{\Gamma_{S}} \times( \pm \vec{z})\right)
$$

the $\rho$ component of (13) is:

$$
\left.\frac{1}{j \omega \epsilon_{0}}\left(\frac{1}{\rho} \frac{\partial H_{z}}{\partial \theta}-\frac{\partial H_{\theta}}{\partial z}\right)\right|_{\Gamma_{S}}= \pm\left. Z_{S} H_{\theta}\right|_{\Gamma_{S}}
$$

due to the $\theta$ invariance, condition (14) becomes:

$$
\left.\frac{\partial H_{\theta}}{\partial z}\right|_{\Gamma_{S}}=\left\{\begin{array}{l}
-\left.j \omega \epsilon_{0} Z_{S} H_{\theta}\right|_{\Gamma_{S}} \text { if } \vec{n}=\vec{z} \\
\left.j \omega \epsilon_{0} Z_{S} H_{\theta}\right|_{\Gamma_{S}} \text { if } \vec{n}=-\vec{z}
\end{array}\right.
$$

so:

$$
\nabla H_{\theta} \cdot \vec{n}=-\left.j \omega \epsilon_{0} Z_{s} H_{\theta}\right|_{\Gamma_{S}}
$$

- for horizontal walls:

$$
\nabla H_{\theta} \cdot \vec{n}= \pm \frac{\partial H_{\theta}}{\partial \rho}
$$

with Maxwell's equation and (1):

$$
\frac{1}{j \omega \epsilon_{0}} \nabla \times\left.\vec{H}\right|_{\Gamma_{S}}=Z_{S}\left(\left.\vec{H}\right|_{\Gamma_{S}} \times( \pm \vec{\rho})\right)
$$

the $z$ component of (18) is:

$$
\left.\frac{1}{j \omega \epsilon_{0}}\left(\frac{1}{\rho}\left(\frac{\partial\left(\rho H_{\theta}\right)}{\partial \rho}-\frac{\partial H_{\rho}}{\partial \theta}\right)\right)\right|_{\Gamma_{S}}=\left.\mp Z_{S} H_{\theta}\right|_{\Gamma_{S}}
$$

where $\rho=\rho_{\text {hor }}$ is the horizontal walls radius.

Due to the $\theta$ invariance, condition (19) becomes:

$$
\left.\frac{\partial H_{\theta}}{\partial \rho}\right|_{\Gamma_{S}}=\left\{\begin{array}{l}
\left.\left(-j \omega \epsilon_{0} Z_{S}-\frac{1}{\rho_{\text {hor }}}\right) H_{\theta}\right|_{\Gamma_{S}} \text { if } \vec{n}=\vec{\rho} \\
\left.\left(j \omega \epsilon_{0} Z_{S}-\frac{1}{\rho_{\text {hor }}}\right) H_{\theta}\right|_{\Gamma_{S}} \text { if } \vec{n}=-\vec{\rho}
\end{array}\right.
$$

so:

$$
\nabla H_{\theta} \cdot \vec{n}=\left.\left(-j \omega \epsilon_{0} Z_{s}-\frac{\vec{n} \cdot \vec{\rho}}{\rho_{\text {hor }}}\right) H_{\theta}\right|_{\Gamma_{s}}
$$

therefore the formulation of (8) with conditions (15) and (20) is:

$$
\begin{gathered}
\iint_{S}\left(\nabla H_{\theta} \cdot \overline{\nabla v}-\left(k_{0}^{2}-\frac{1}{\rho^{2}}\right) H_{\theta} \bar{v}\right) \rho d \rho d z \\
+\sum_{\Gamma_{\text {vert }}}\left(j \omega \epsilon_{0} Z_{S}\right) \int_{\Gamma_{\text {vert }}} \bar{v} H_{\theta} \rho d \rho \\
+\sum_{\Gamma_{\text {hor }}}\left(j \omega \epsilon_{0} Z_{S} \rho_{\text {hor }}+\vec{n} \cdot \vec{\rho}\right) \int_{\Gamma_{\text {hor }}} \bar{v} H_{\theta} d z=0 .
\end{gathered}
$$

To solve (22) the linear system (23) is implemented:

$$
A^{H} \vec{H}_{\theta}=\overrightarrow{0}
$$

where $A^{H}=\left[A_{i j}^{H}\right]$ and $\vec{H}_{\theta}$ is defined as follows [20]:

$$
\vec{H}_{\theta}=\left[\begin{array}{c}
u_{1} \\
\vdots \\
u_{N}
\end{array}\right], \quad H_{\theta}=\sum_{j=1}^{N} u_{j} \Phi_{j}
$$

where $N$ is the number of nodes, $u_{j}$ is the value of $H_{\theta}$ at the node $j$ in the triangular basis of $\Phi_{i}$ and $\Phi_{i}$ the basis function of the vector space. Hence: 


$$
\begin{aligned}
& A_{i j}^{H}=\iint_{S}\left(\nabla \Phi_{j} \cdot \overline{\nabla \Phi}_{i}-\left(k_{0}^{2}-\frac{1}{\rho^{2}}\right) \Phi_{j} \bar{\Phi}_{i}\right) \rho d \rho d z \\
& \quad+\sum_{\Gamma_{\text {vert }}}\left(j \omega \epsilon_{0} Z_{S}\right) \int_{\Gamma_{\text {vert }}} \bar{\Phi}_{i} \Phi_{j} \rho d \rho \\
& \quad+\sum_{\Gamma_{\text {hor }}}\left(j \omega \epsilon_{0} Z_{S} \rho_{\text {hor }}+\vec{n} \cdot \vec{\rho}\right) \int_{\Gamma_{\text {hor }}} \bar{\Phi}_{i} \Phi_{j} d z .
\end{aligned}
$$

If $\Gamma_{S}$ is a Perfect Electric Conductor $Z_{S}=0,(25)$ becomes:

$$
\begin{array}{r}
A_{i j}^{H}=\iint_{S}\left(\nabla \Phi_{j} . \bar{\nabla}_{i}-\left(k_{0}^{2}-\frac{1}{\rho^{2}}\right) \Phi_{j} \bar{\Phi}_{i}\right) \rho d \rho d z \\
+\sum_{\Gamma_{\text {hor }}} \vec{n} . \vec{\rho} \int_{\Gamma_{h o r}} \bar{\Phi}_{i} \Phi_{j} d z
\end{array}
$$

(25) or (26) is solved to obtain $H_{\theta}$ the magnetic field component (24). The surface impedance in the plane $\Omega^{\prime}$ means a computation of the electric field component $E_{z}$. From Maxwell equations:

$$
\nabla \times \vec{H}=j \omega \epsilon_{0} \vec{E}
$$

therefore:

$$
\left.E_{z}\right|_{\Omega^{\prime}}=\frac{1}{j \omega \epsilon_{0}}\left(\frac{\left.H_{\theta}\right|_{\Omega^{\prime}}}{a}+\left.\frac{\partial H_{\theta}}{\partial \rho}\right|_{\Omega^{\prime}}\right)
$$

consequently the surface impedance in $\Omega^{\prime}$ is:

$$
Z_{Z}^{\prime}=\left.\frac{E_{Z}}{H_{\theta}}\right|_{\Omega^{\prime}}=\frac{1}{j \omega \epsilon_{0}}\left(\frac{1}{a}+\frac{\left.\frac{\partial H_{\theta}}{\partial \rho}\right|_{\Omega^{\prime}}}{\left.H_{\theta}\right|_{\Omega^{\prime}}}\right)
$$

\section{3) The Characterization of $Z_{T}^{\prime}$}

$Z_{T}^{\prime}$ defined in (2) is generated by $E_{\theta}$ which is considered $\theta$ invariant. $E_{\theta}$ is also solution of (8).

The same calculation steps as the Section II.B.2) are carried out to obtain the formulation for the electric field:

$$
\begin{gathered}
\iint_{S}\left(\nabla E_{\theta} \cdot \overline{\nabla v}-\left(k_{0}^{2}-\frac{1}{\rho^{2}}\right) E_{\theta} \bar{v}\right) \rho d \rho d z \\
+\sum_{\Gamma_{v e r t}}\left(\frac{j \omega \mu_{0}}{Z_{S}}\right) \int_{\Gamma_{v e r t}} \bar{v} E_{\theta} \rho d \rho \\
+\sum_{\Gamma_{\text {hor }}}\left(\frac{j \omega \mu_{0} \rho_{\text {hor }}}{Z_{S}}+\vec{n} . \vec{\rho}\right) \int_{\Gamma_{\text {hor }}} \bar{v} E_{\theta} d z=0 .
\end{gathered}
$$

To solve (30) the linear system (31) is implemented:

$$
A^{E} \vec{E}_{\theta}=\overrightarrow{0}
$$

where $A^{E}=\left[A_{i j}^{E}\right]$ and $\vec{E}_{\theta}$ is defined as follows:

$$
\vec{E}_{\theta}=\left[\begin{array}{c}
u_{1} \\
\vdots \\
u_{N}
\end{array}\right], \quad E_{\theta}=\sum_{j=1}^{N} u_{j} \Phi_{j}
$$

where $N$ is the number of nodes, $u_{j}$ is the value of $E_{\theta}$ at the node $j$ in the triangular basis of $\Phi_{i}$ and $\Phi_{i}$ the basis function of the vector space. It follows that:

$$
\begin{aligned}
A_{i j}^{E}= & \iint_{S}\left(\nabla \Phi_{j} \cdot \overline{\nabla \Phi}_{i}-\left(k_{0}^{2}-\frac{1}{\rho^{2}}\right) \Phi_{j} \bar{\Phi}_{i}\right) \rho d \rho d z \\
& +\sum_{\Gamma_{\text {vert }}}\left(\frac{j \omega \mu_{0}}{Z_{S}}\right) \int_{\Gamma_{\text {vert }}} \bar{\Phi}_{i} \Phi_{j} \rho d \rho \\
& +\sum_{\Gamma_{\text {hor }}}\left(\frac{j \omega \mu_{0} \rho_{\text {hor }}}{Z_{S}}+\vec{n} . \vec{\rho}\right) \int_{\Gamma_{\text {hor }}} \bar{\Phi}_{i} \Phi_{j} d z
\end{aligned}
$$

when $\Gamma_{s}$ is a PEC:

$$
\left.E_{\theta}\right|_{\Gamma_{S}}=0
$$

therefore (33) becomes:

$A_{i j}^{E}=\iint_{S}\left(\nabla \Phi_{j} \cdot \overline{\nabla \Phi}_{i}-\left(k_{0}^{2}-\frac{1}{\rho^{2}}\right) \Phi_{j} \bar{\Phi}_{i}\right) \rho d \rho d z=0$

(33) or (35) is solved to obtain $E_{\theta}$ the electric field component. The surface impedance in the plane $\Omega^{\prime}$ means a computation of the magnetic field component $H_{z}$. From Maxwell's equations:

$$
\nabla \times \vec{E}=-j \omega \mu_{0} \vec{H}
$$

therefore:

$$
\left.H_{z}\right|_{\Omega^{\prime}}=\frac{j}{\omega \mu_{0}}\left(\frac{\left.E_{\theta}\right|_{\Omega^{\prime}}}{a}+\left.\frac{\partial E_{\theta}}{\partial \rho}\right|_{\Omega^{\prime}}\right)
$$

consequently the surface impedance in $\Omega^{\prime}$ is:

$$
Z_{T}^{\prime}=-\left.\frac{E_{\theta}}{H_{Z}}\right|_{\Omega^{\prime}}=-\frac{j \omega \mu_{0}}{\frac{1}{a}+\frac{\left.\frac{\partial E_{\theta}}{\partial \rho}\right|_{\Omega^{\prime}}}{\left.E_{\theta}\right|_{\Omega^{\prime}}}}
$$

\section{The Recursive Solution of Dispersion Equation}

The surface impedances $\left(Z_{T}^{\prime}\right.$ and $\left.Z_{Z}^{\prime}\right)$ depend on the incidence angle [18]. In addition in cylindrical waveguides this angle should be taken into consideration. Fig. 3 shows the components of the propagation constants: 


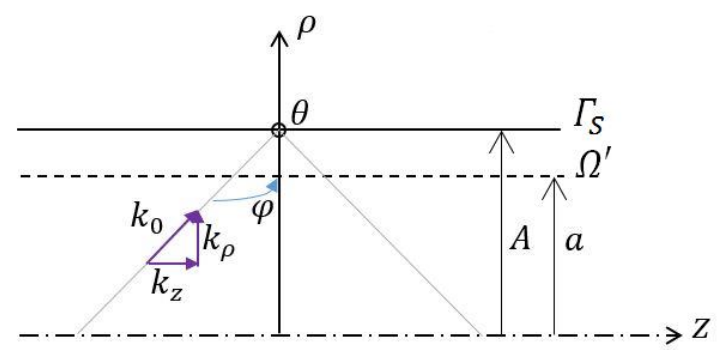

Fig. 3. Electromagnetic wave propagation in a half-plane $(\rho 0 z)$ of the cylindrical waveguide with the propagation constant components.

$$
\overrightarrow{k_{0}}=\left\{\begin{array}{l}
k_{\rho} \\
k_{\theta} \\
k_{z}
\end{array}=\left\{\begin{array}{c}
k_{0} \cos \varphi \\
0 \\
k_{0} \sin \varphi
\end{array}\right.\right.
$$

where $\varphi$ is the incidence angle.

Hence the $\varphi$ angle is deduced from $k_{z}$ :

$$
\varphi=\operatorname{asin}\left(\frac{k_{z}}{k_{0}}\right)
$$

where $k_{z}$ is the phase constant along the $z$-axis.

The algorithm in Fig. 4 is implemented to compute the dispersion diagrams.

The FEM code is necessary to compute the conformal surface impedances. With this algorithm, different 2D metamaterials (with $\theta$ invariance) in cylindrical waveguides can be processed. A 3D FEM code will be required to deal with 3D metamaterials or modes with angular dependency $(m$ order different from 0 ).

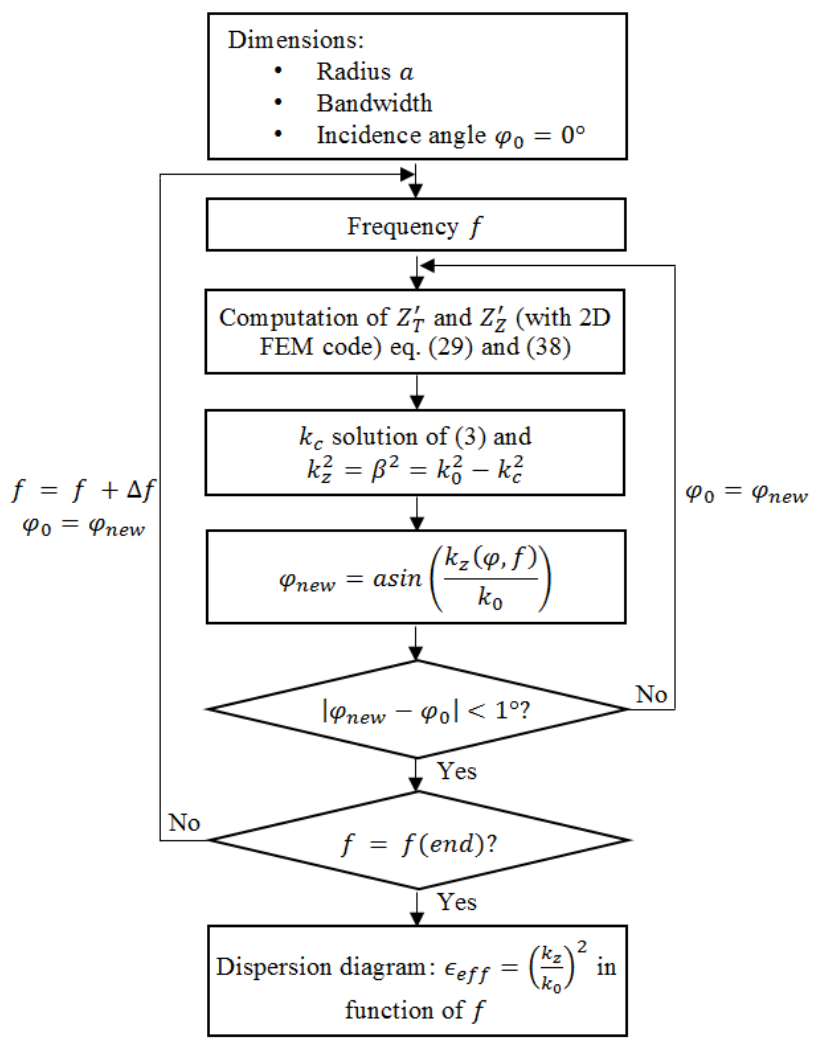

Fig. 4. Schematic algorithm to correct the angle $\varphi$.

\section{RESULTS}

In this Section, the method and algorithms are validated in three different cases:

- firstly the values of the $Z_{S}$ surface impedance $\left(Z_{T}\right.$, $Z_{Z}$ ) is fixed at the $A$ radius. Dispersion diagrams of these waveguides are obtained with the algorithm of [16]. A comparison is made with dispersion diagrams computed with the algorithm presented Fig. 4 and the $Z_{S}^{\prime}$ surface impedances $\left(Z_{T}^{\prime}, Z_{Z}^{\prime}\right)$ evaluated for a given $a$ radius,

- $\quad$ subsequently the code is applied to a metamaterial: a corrugation. This result is compared with the result of [15], to show the improvements made thanks to the 2D FEM code,

- finally the code is applied to a T-structure metamaterial.

\section{A. Cylindrical Waveguides with Isotropic and Anisotropic Surface Impedances}

Initially to validate the method, the FEM code is applied for the fixed $\left(Z_{T}, Z_{Z}\right)$ surface impedances at the $A$ radius. The FEM code computes the $\left(Z_{T}^{\prime}, Z_{Z}^{\prime}\right)$ impedances at another $a$ radius. The unit cell is created with the open source software Gmsh [21] and represented in Fig. 5.

(22) and (30) can be simplified, as there is only one horizontal wall, therefore $H_{\theta}$ is a solution of:

$$
\begin{aligned}
\iint_{S}\left(\nabla H_{\theta} \cdot \overline{\nabla v}-(\right. & \left.\left.k_{0}^{2}-\frac{1}{\rho^{2}}\right) H_{\theta} \bar{v}\right) \rho d \rho d z \\
& +\left(j \omega \epsilon_{0} Z_{S} A-1\right) \int_{\Gamma_{S}} H_{\theta} \bar{v} d z=0
\end{aligned}
$$

$E_{\theta}$ is a solution of:

$$
\begin{aligned}
\iint_{S}\left(\nabla E_{\theta} \cdot \overline{\nabla v}-(\right. & \left.\left.k_{0}^{2}-\frac{1}{\rho^{2}}\right) E_{\theta} \bar{v}\right) \rho d \rho d z \\
& +\left(\frac{j \omega \mu_{0} A}{Z_{S}}-1\right) \int_{\Gamma_{S}} E_{\theta} \bar{v} d z=0 .
\end{aligned}
$$

The dispersion diagrams obtained for a cylindrical waveguide of radius $\mathrm{A}=30 \mathrm{~mm}$ and $a=28 \mathrm{~mm}$ are illustrated for various anisotropic surface impedances (curves with dots). The results are compared with the dispersion diagrams obtained with the code presented in [16] (curves with circles) in Fig. 6. The dispersion diagrams are identical hence the elaborated algorithm is validated.

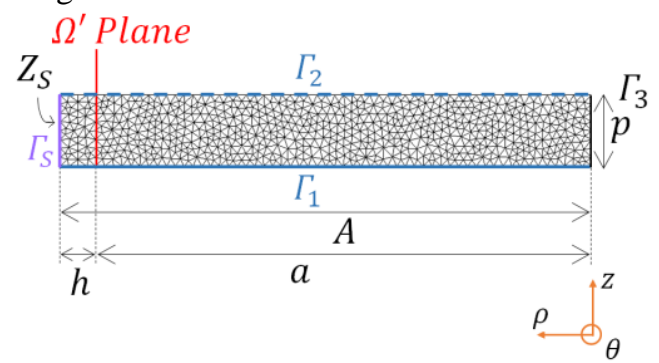

Fig. 5. Unit cell mesh and dimensions: $A=30 \mathrm{~mm}, a=28 \mathrm{~mm}, h=2 \mathrm{~mm}$, $p=4 \mathrm{~mm}$ 


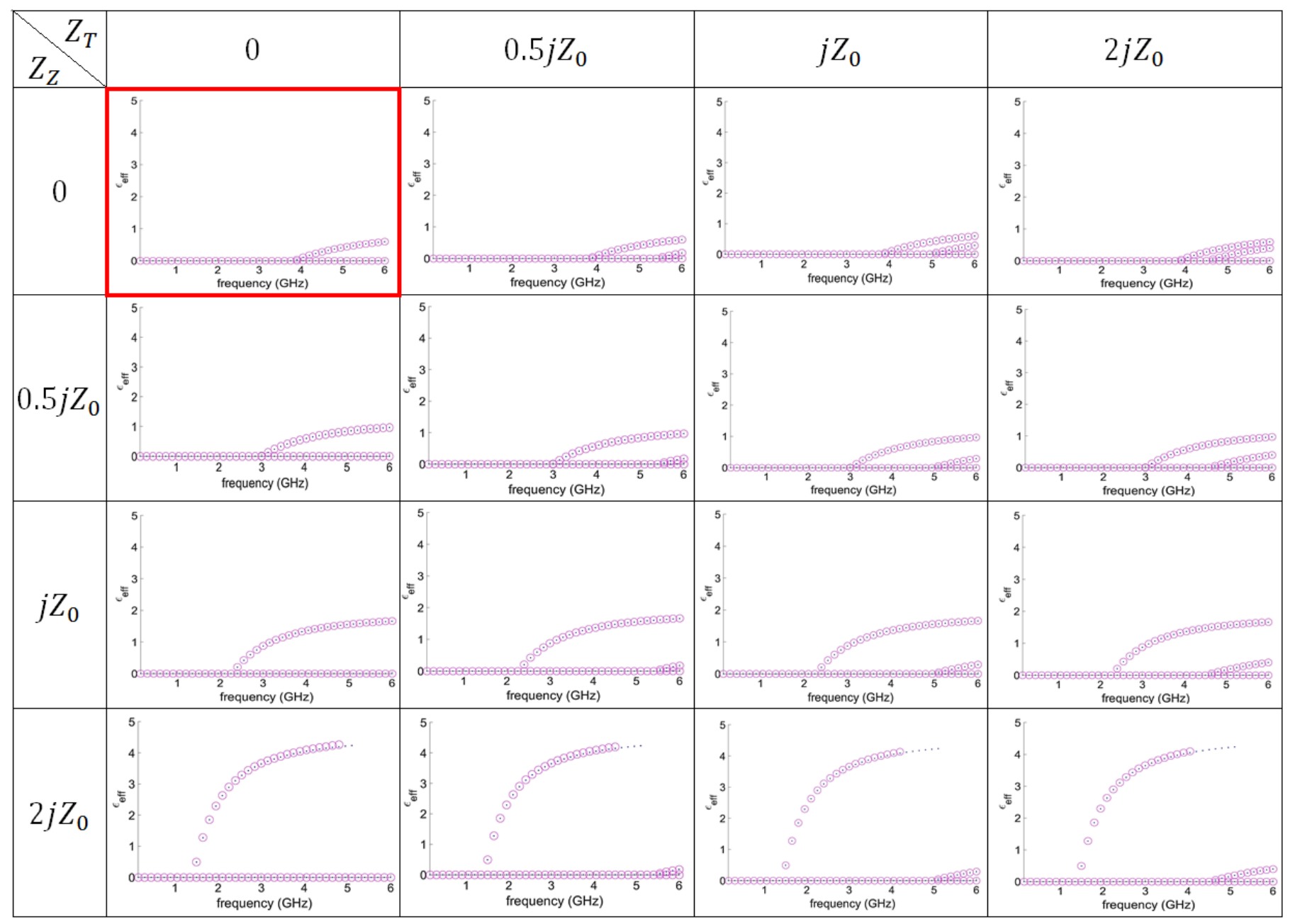

Fig. 6. Dispersion diagrams of a cylindrical waveguide for various anisotropic surface impedances $\left(Z_{T}, Z_{Z}\right)$. The curves with dots are obtained with the code which computes the $\left(Z_{T}^{\prime}, Z_{Z}^{\prime}\right)$ impedances at a certain distance $h$ (here $h=2 \mathrm{~mm}$ ) and the curves with circles are obtained with the code based on MET [16].

\section{B. A Corrugated Cylindrical Waveguide}

A corrugation invariant in $\theta$ direction is now considered. Fig. 7 presents the corrugated waveguide.

To study this waveguide with HFSS, the same method as [16] was used. Dispersion diagrams are obtained from the section represented in Fig. 6 with dashes and using periodic boundary conditions, see Fig. 8 . The propagation constant is computed thanks to the phase delay between two periodic boundary conditions and the distance between them. By using the eigenmode solver in HFSS, the frequency of each solution is given. The complete dispersion diagram is obtained by varying the phase delay between the two periodic boundary conditions from $0^{\circ}$ to $180^{\circ}$.

To study this waveguide with the MET, the section of the waveguide used is extracted in Fig. 7 with dots. Whereas the structure in HFSS is in 3D, for the MET only a 2D representation is useful. The unit cell is represented in Fig. 9. A 2D waveguide section cannot be studied with HFSS. For a MET validation purpose, a comparison is done with this software. Since the MET is in 2D and HFSS in 3D, the computation time is drastically improved anytime with the MET.

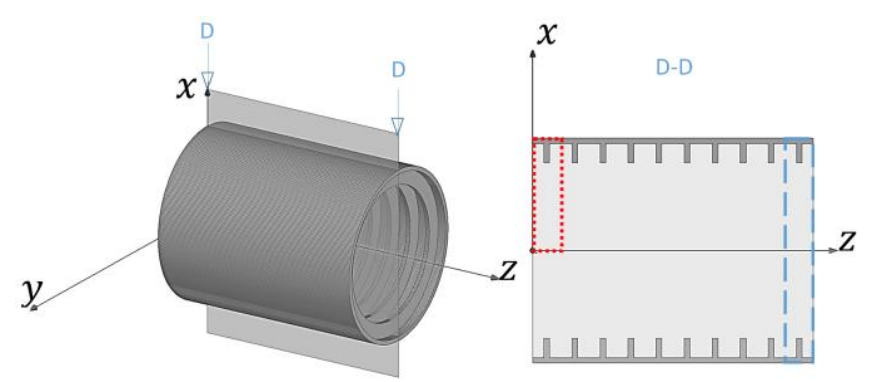

Fig. 7. Corrugated waveguide. The dashed section is the section used in HFSS and the dotted part is used in the MET.

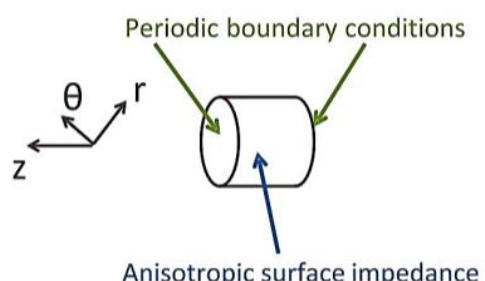

Fig. 8. Cylindrical representation of a waveguide with periodic boundary conditions and anisotropic surface simulated in HFSS. 


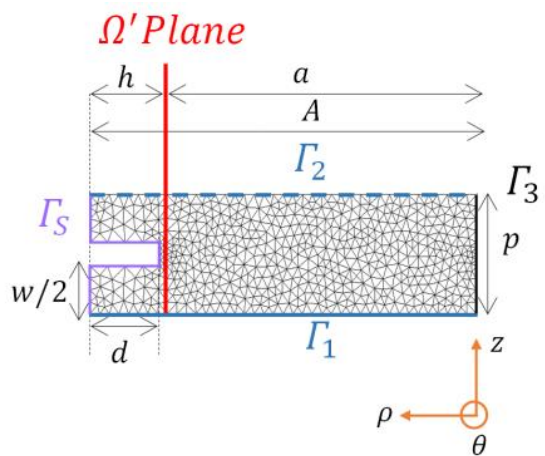

Fig. 9. Unit cell mesh and dimensions: $A=100 \mathrm{~mm}, a=80 \mathrm{~mm}, h=$ $20 \mathrm{~mm}, p=26.225 \mathrm{~mm}, w=20.98 \mathrm{~mm}$ and $d=18.2 \mathrm{~mm}$.

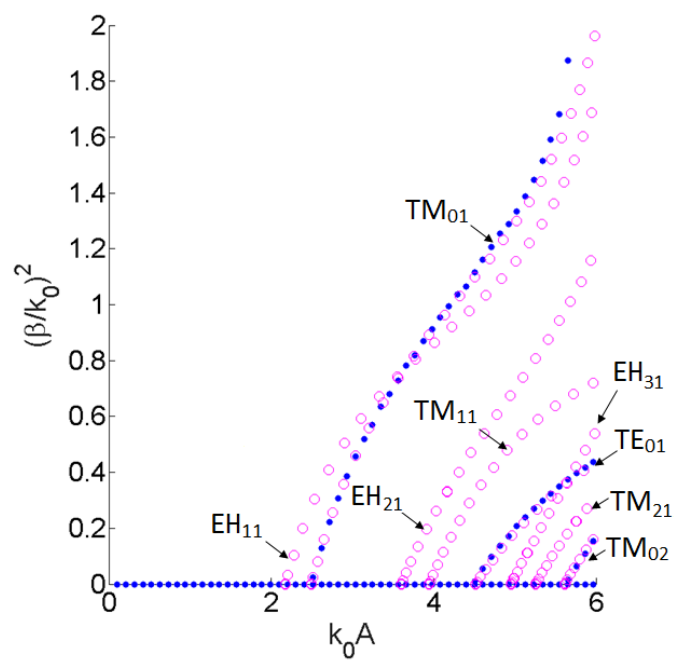

Fig. 10. Dispersion diagrams of the cylindrical waveguide with the corrugation presented in Fig. 7 obtained with MET (dots) and HFSS (circles).

For the corrugation $\Gamma_{S}$ is a PEC hence (24) and (42) are solved. The dispersion diagrams obtained are compared with HFSS results in Fig. 10. Both diagrams perfectly coincide with each other. There is a clear improvement compared to the dispersion diagram in [15]. Using HFSS the dispersion diagram is obtained in three days of simulation (Intel (R) Xeon (R), $1.8 \mathrm{GHz} 2$ processors, $64 \mathrm{~GB}$ of RAM). Using the MET the dispersion diagram requires a twelve-minute computation time. Consequently the dispersion diagram is obtained with the MET around 360 times faster than with HFSS thanks to the 2D resolution.

\section{C.A T-Structure Metamaterial Waveguide}

A T-structure invariant in $\theta$ direction is studied. In Fig. 11 the waveguide is presented. The dashed section represented in Fig. 11 is extracted and simulated in a 3D FEM code (HFSS) while the dotted section is inserted in the 2D FEM code in the MET. The unit cell and the dimensions of this section are represented in Fig. 12.
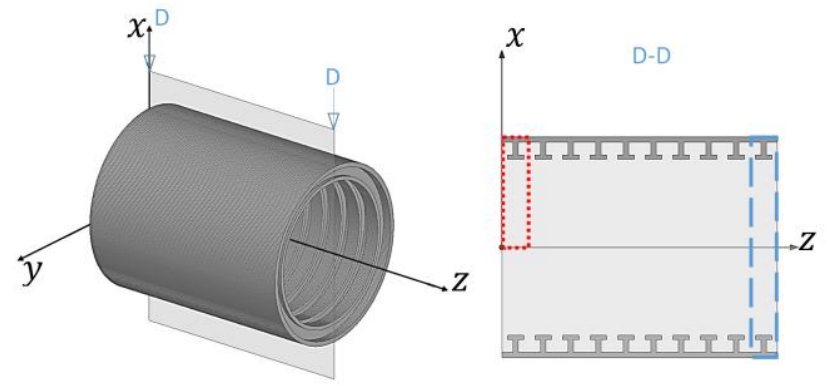

Fig. 11. Waveguide with T-structure metamaterial. The dashed section is the section used in HFSS and the dotted section is used in the MET.

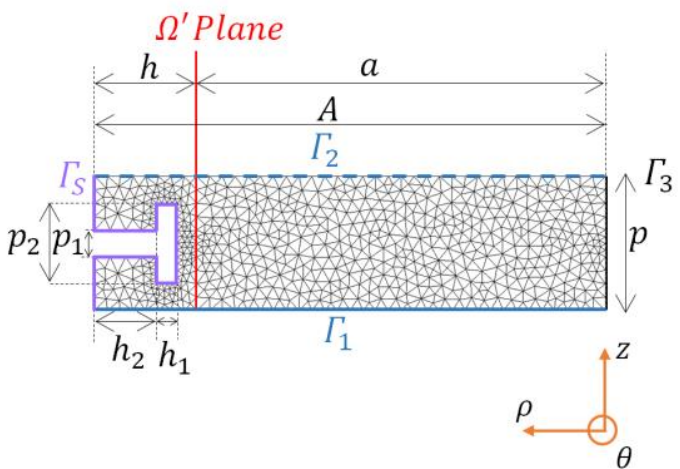

Fig. 12. Unit cell mesh and dimensions: $A=50 \mathrm{~mm}, a=40 \mathrm{~mm}, h=$ $10 \mathrm{~mm}, \quad h_{1}=2.025 \mathrm{~mm}, \quad h_{2}=6.075 \mathrm{~mm}, \quad p=13.1125 \mathrm{~mm}, \quad p_{1}=$ $2.6225 \mathrm{~mm}$, and $p_{2}=7.8675 \mathrm{~mm}$.

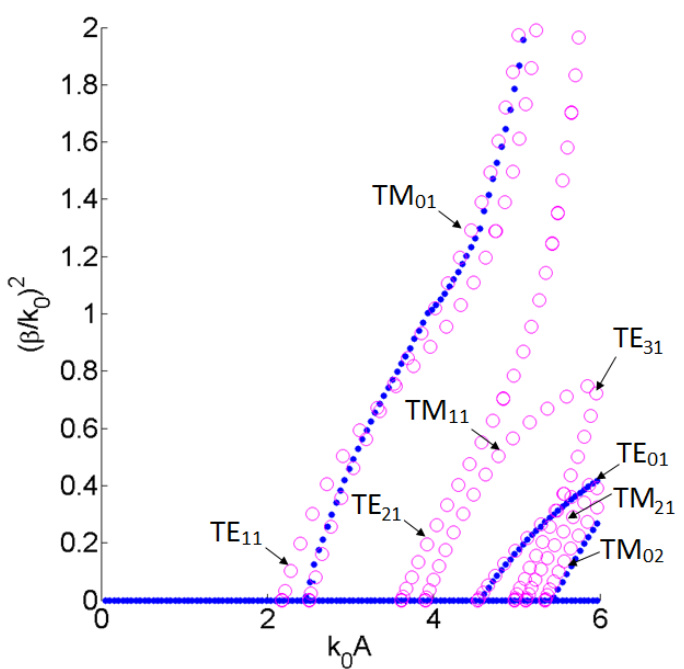

Fig. 13. Dispersion diagrams of the cylindrical waveguide with the T-structure presented in Fig. 11 obtained with MET (dots) and HFSS (circles).

As in the previous case, $\Gamma_{S}$ is a PEC, so (24) and (42) are solved. The dispersion diagrams obtained are compared with the HFSS result in Fig. 13. The simulation time (Intel (R) Xeon (R), $1.8 \mathrm{GHz} 2$ processors, $64 \mathrm{~GB}$ of RAM) with the MET code is 31 minutes compared to the $3 \mathrm{D}$ simulation in HFSS which lasts almost four days.

With this new code it is possible to obtain the dispersion diagram for $m=0$ modes for all kinds of cylindrical waveguides with 2D metamaterial invariant along the $\theta$-axis. 
To have the dispersion diagrams with all the modes, the same algorithm can be used, however another MET code should be developed using 3D FEM.

\section{CONCLUSION}

The Modal Expansion Theory has been developed to compute the dispersion properties of cylindrical waveguides with 2D metamaterials for 0 order modes. To demonstrate the accuracy and the time efficiency of the new method three different waveguides have been studied: One waveguide with fixed surface impedances and two with metamaterials which are invariant in $\theta$ direction. The MET method allows the characterization of isotropic and anisotropic waveguides properties whereas HFSS characterizes only isotropic waveguides. Furthermore all the dispersion diagrams are obtained in less than a ten-minute computation time while this process takes more than one hour by using HFSS, as a 3D Finite Element Method resolution is used in the commercial software while in the MET it is a 2D FEM resolution. Consequently the time efficiency of the MET is more relevant with 2D metamaterials. Indeed for the corrugated waveguide, the time needed for characterization of propagation properties is divided by 360 with respect to HFSS. As for the T-structure waveguide, the time is divided by almost 200 .

The more complicated the structure, the more time is saved. This method is now under development for 3D metamaterials, to deal with all the mode orders and all other potential metamaterial structures. Since structures can vary with $\theta$ the 3D FEM code cannot be simplified into 2D FEM code anymore.

\section{REFERENCES}

[1] R. A. Shelby, D. R. Smith and S. Schultz, "Experimental Verification of a Negative Index of Refraction," Sci., vol. 292, no. 5514, pp. 77-79, Apr. 2001.

[2] D. R. Smith, W. J. Padilla, D. C. Vier, S. C. NematNasser, and S. Schultz, "Composite Medium with Simultaneoustly Negative Permeability and Permittivity," Phys. Rev. Lett., vol. 84, no. 18, pp. 41844187, May 2000.

[3] V. G. Veselago, "The Electrodynamics of Substances with Simultaneously Negative Values of $\varepsilon$ and $\mu$," Sov. Phys. Usp., vol. 10, no. 4, pp. 509-514, 1968.

[4] R. W. Ziolkowski and E. Heyman, "Wave Propagation in Media Having Negative Permittivity and Permeability," Phys. Rev. E., vol. 64, no. 5, pp. 1-15, 2001, Art. ID 056625 .

[5] Q. Wu, M. D. Gregory, D. H. Werner, P. L. Werner and E. Lier, "Nature-Inspired Design of Soft, Hard and Hybrid Metasurfaces," Proc. 2010 IEEE Int. Symp. on Antennas and Propagation and USNC/URSI National Radio Science Meeting, Toronto, Canada, July 11-17, 2010, pp. 1-4.
[6] J. G. Pollock and A. K. Iyer, "Below-Cutoff Propagation in Metamaterial-Lined Circular Waveguides," IEEE Trans. Microw. Theory Techn., vol. 61, no. 9, pp. 31693178, Sep. 2013.

[7] J. G. Pollock and A. K. Iyer, "Radiation Characteristics of Miniaturized Metamaterial-Lined Waveguide Probe Antennas," Proc. 2015 IEEE Int. Symp. on Antennas and Propagation and USNC/URSI National Radio Science Meeting, Vancouver, Canada, July 2015.

[8] J. G. Pollock and A. K. Iyer, "Miniaturized CircularWaveguide Probe Antennas using Metamaterial Liners," IEEE Trans. Antennas Propag., vol. 63, no. 1, pp. 428433, Jan. 2015.

[9] J. G. Pollock and A. K. Iyer, "Experimental Verification of Below-Cutoff Propagation in Miniaturized Circular Waveguides Using Anisotropic ENNZ Metamaterial Liners," IEEE Trans. Microw. Theory Techn., vol. 64, no. 4, pp. 1297-1305, Apr. 2016.

[10] P.-S. Kildal and E. Lier, "Hard Horns Improve Cluster Feeds of Satellite Antennas,» Electronics Letters, vol. 24, no. 8, pp. 491-492, Apr. 1988.

[11] R. K. Shaw, E. Lier and C.-C. Hsu., «Profiled Hard Metamaterial Horns for Multibeam Reflectors," Proc. 2010 IEEE International Symposium on Antennas and Propagation and USNC/URSI National Radio Science Meeting, Toronto, Canada, July 11-17, 2010, pp. 1-4.

[12] H. Minnett. and B. Thomas, "A Method of Synthesizing Radiation Patterns with Axial Symmetry," IEEE Trans. Antennas Propag., vol. 14, no. 5, pp. 654-656, Sep. 1966.

[13] E. Lier, "Review of Soft and Hard Horn Antennas, Including Metamaterial-Based Hybrid-Mode Horns," IEEE Antennas Propag. Mag., vol. 52, no. 2, pp. 31-39, Apr. 2010.

[14] B. Byrne, N. Raveu, N. Capet, G. Le Fur and L. Duchesne, "Reduction of Rectangular Waveguide CrossSection with Metamaterials: A New Approach," presented at the 9th Int. Congr. on Advanced Electromagnetic Materials in Microwaves and Optics Metamaterials, Oxford, United Kingdom, Sep. 7-12, 2015, pp. 40-42.

[15] B. Byrne, N. Capet and N. Raveu, "Dispersion Properties of Corrugated Waveguides Based on the Modal Theory," in Proc. 8th Eur. Conf. on Antennas Propag., The Hague, Netherland, Apr. 6-11, 2014, pp.1-3.

[16] N. Raveu, B. Byrne, L. Claudepierre and N. Capet, "Modal Theory for Waveguides with Anisotropic Surface Impedance Boundaries," IEEE Trans. Microw. Theory Techn., vol. 64, no. 4 pp. 1153-1162, Apr. 2016.

[17] B. Byrne, "Etude et Conception de Guides d'Onde et d'Antennes Cornets à Métamatériaux," These de doctorat d'état, Univ. Toulouse, Toulouse, 2016.

[18] B. Byrne, N. Raveu, N. Capet, G. Le Fur and L. Duchesne, "Modal analysis of Rectangular Waveguides with 2D Metamaterials," Progress In Electromagnetics Research C, vol. 70, pp. 165-173, 2016. 
[19] P. Lacoste, "Solution of Maxwell Equation in Axisymmetric Geometry by Fourier Series Decomposition and by Use of H(rot) Conforming Finite Element," Numerische Mathematik, vol. 84, pp. 577-609, 2000.

[20] J.-M. Jin, The Finite Element Method in Electromagnetics, 3rd ed. Hoboken (New Jersey), USA: Wiley, 2014.

[21] C. Geuzaine, and J.-F. Remacle "Gmsh: A threedimensional finite element mesh generator with built-in pre- and post-processing facilities," International Journal for Numerical Methods in Engineering, vol. 79, no. 111, pp. 1309-1331, 2009.

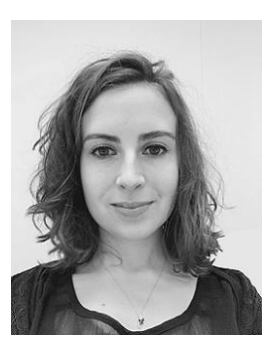

Lucille Kuhler was born in Metz, France, in 1992. She received the degree in electronic engineering and hyperfrequencies from the Ecole National d'Aviation Civile (ENAC), Toulouse, France, in 2016. Currently she is working toward the $\mathrm{Ph}$. D. degree in electromagnetism and microwaves at the University of Toulouse, Toulouse,

France

Her research topics are electromagnetic wave propagation, antennas, and metamaterials.

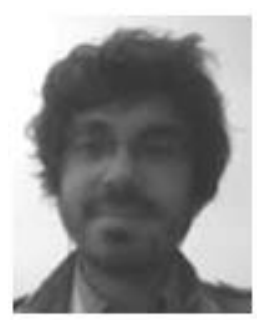

Gwenn Le Fur received the M.S. degree in signal processing and telecommunications from the University of Rennes 1, Rennes, France, in 2006 and the Ph.D. degree from the Rennes Institute of Electronics and Telecommunications, Rennes, France, in 2009.

He was first with the CEA LETI, Grenoble, France, where he worked on characterization of small antenna using noninvasive measurement methods and integrated multi-antenna system. Then he joined SATIMO Industries (Microwave Vision Group) as a R\&D Engineer. He is now with the Centre National d'Etudes Spatiales (CNES), Toulouse, France, working on antenna measurement techniques.

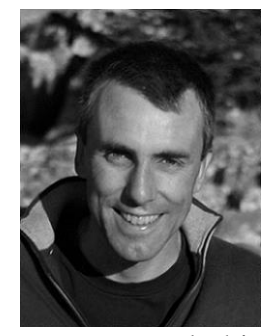

Luc Duchesne got his diploma of engineer in electronics in 1992 from Ecole Supérieure d'Electronique de l'Ouest (ESEO), Angers, France and then a specialization in aerospace electronics in 1994 from Ecole Nationale Supérieure de l'Aéronautique et de 1'Espace (ISAE Supaéro), Toulouse, France.

He started his professional life in 1994 at Deutsche Aerospace (Dasa) in Munich (now Airbus Defence and Space) as an Antennas and Payload Components Engineer.

In August 2000, he joined SATIMO close to Paris (Now Microwave Vision) as Research and Development Director.
From 2000 on to present, he participated to build a strong R\&D department within SATIMO dedicated to the development of innovative products for the core activity of antenna measurement systems but also for new activities concerning non-destructive testing systems and imagery systems.

He co-authored several conference papers about antennas and antenna measurement systems. He is also co-inventor of several patents at DASA and SATIMO.

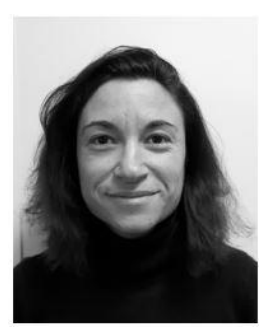

Nathalie Raveu received the M.S. degree in electronics and signal processing in 2000 and the Ph. D. degree in 2003.

She is a Professor with the National Polytechnic Institute of Toulouse (INPT) and a Research Fellow with the LAPLACE-CNRS (LAboratory of PLAsma and Energy Conversion). Her research topics are oriented toward development of efficient numerical techniques to address innovative microwave circuits. During the last years, she has developed a new method for SICs study, metamaterial horns, and plasma cavity. 